\title{
A cientificidade das técnicas projetivas em debate
}

\author{
Anna Elisa de Villemor-Amaral \\ Lílian Pasqualini-Casado²
}

\begin{abstract}
Resumo
O objetivo deste trabalho é rever uma série especial de artigos antagônicos sobre a validade e o status científico dos métodos projetivos, uma vez que o foco dos debates atuais recai novamente sobre o mérito das técnicas projetivas em contraposição às objetivas, principalmente no que diz respeito ao pouco rigor metodológico nos estudos de validação e ausência de validade incremental, o que, conseqüentemente, repercute na credibilidade dos profissionais e pesquisadores que as utilizam. Neste artigo, dá-se destaque ao que há de procedente em tais discussões, bem como nas distorções ou falácias geradas nesta última década sobre os três procedimentos mais utilizados para avaliação da personalidade, no Brasil e exterior: o Método de Rorschach no Sistema Compreensivo, RCS; o Teste de Apercepção Temática, TAT e o Desenho da Figura Humana, DFH. Os referenciais apresentados sobre a utilidade clínica e diagnóstica desses instrumentos mostram-se igualmente úteis na ponderação de estudos e na discussão de questões relativas às técnicas projetivas de modo geral.

Palavras-chave: Avaliação psicológica; Métodos projetivos; Rorschach; Teste de Apercepção Temática; Desenho da Figura Humana; Validade.
\end{abstract}

\section{The scientific status of projective techniques in discussion}

\begin{abstract}
The aim of this paper is to review and discuss a special series of antagonistic articles about the scientific status of the projective methods for personality assessment since the focus of the current debates has fallen again into the merit of the projectives in contraposition to the objective techniques. The critics are mainly in respect to the little methodologic procedures in the validation studies as well the absence of incremental validity for the projective methods, what consequently reverberates in the credibility of the professionals and researchers who use them. In this circumstances, this work emphasizes the positive aspects on the controversial as well the distortions or fallacies generated in this last decade about the three used procedures for personality assessment, in Brazil and exterior: the Method of Rorschach in the Comprehensive System, RCS; Aperception Thematic Test, TAT and the Drawing of the Figure Human being, DFH. The questions presented on the clinical and diagnostic utility of these instruments reveal equally useful in the balance of studies and the quarrel of relative questions the projective techniques in general way.
\end{abstract}

Keywords: Psychological assessment; Projective methods; Rorschach SC; Aperception Thematic Test; DFH; Validity.

Revendo-se a literatura, é possível constatar que são cíclicos os períodos de críticas aos procedimentos aplicados no campo da Avaliação Psicológica e que determinadas épocas destacam-se pelo ceticismo ou mesmo pela total negação da importância dos métodos e técnicas de um modo geral.

Quanto aos métodos projetivos, se nas décadas de 60 e 70 observou-se a emergência de controvérsias generalizadas sobre sua natureza e utilidade, bem como severas críticas a uma fase antecedente de idealizações, inconsistências e uso abusivo das mesmas, a partir de então, também têm sido significativas as publicações na área que estabelecem suficiente acúmulo de evidências sobre suas confiabilidades e validades, seus alcances e limitações.

Como em cada período predominam certas tendências e atitudes científicas, após fase de relativa calmaria, o início do século XXI vem carregado de nova onda de preocupações, de acalorados debates sobre o status científico dos métodos de investigação da personalidade. $\mathrm{O}$ foco das discussões recentes está no mérito das técnicas projetivas em contraposição às objetivas, principalmente no que diz respeito ao pouco rigor metodológico nos estudos de validação e à ausência de validade incremental em relação aos primeiros.

Diante das circunstâncias, a proposta deste artigo é destacar o que há de procedente e o que há de distorções ou falácias em tais controvérsias, procurando integrar as informações pertinentes aos três procedimentos mais utilizados no exterior e no Brasil (Herzberg \& Mattar, 2005) para avaliação da personalidade: o Método de Rorschach no Sistema Compreensivo, RCS (Exner, 1991, 1994, 2001, 2003); o Teste de Apercepção Temática, TAT (Murray, 1943,

Endereço para correspondência:

${ }^{1}$ E-mail: aevillemor@terra.com.br

${ }^{2}$ E-mail: lic.pasqualini@uol.com.br 
1995) e o Desenho da Figura Humana, DFH (Machover, 1949; Goodenough, 1926; Harris, 1991).

Antes porém de abordar cada uma das técnicas separadamente, serão assinalados alguns pontos diferenciais dos procedimentos de medidas objetivas e projetivas, ponto inicial das polêmicas.

Instrumentos de medidas projetivas e de medidas objetivas acessam informações em níveis diferentes. Mesmo se designados a medir construtos paralelos; instrumentos de medidas objetivas - como entrevistas e escalas auto-relatos - e de medidas projetivas - como Rorschach, TAT e DFH - apreendem aspectos distintos de um mesmo estado motivacional, traço ou necessidade (McClelland, Koestner \& Weinberg, em Bornstein, 2002; em Meyer \& Handler, 1997). Como necessidades explícitas estão mais relacionadas àquelas motivações que as pessoas reconhecem como características de seus funcionamentos rotineiros, estas podem ser acessadas via medidas objetivas. Assim, escores de medidas auto-atribuídas devem predizer respostas mais intencionais, dadas quase exclusivamente em circunstâncias nas quais as implicações de um motivo ou necessidade especial são claras para quem as relata. Em contraste, espera-se que os escores de medidas projetivas prognostiquem comportamento de tendências espontâneas, subjetivas, motivadas por necessidades implícitas e mais passíveis de tornarem-se manifestas em certos contextos.

O fato de medidas diferentes de um mesmo construto apresentarem uma baixa correlação dos escores não deve ser reconhecido como necessariamente um problema de validade convergente. Em certas situações é justamente a fraca correlação que sustenta evidências de validade a ambas, o que permitirá avaliar de uma outra perspectiva a utilidade clínica e empírica de ambos os métodos para um dado construto (Bornstein, 2001; 2002).

Realmente, os métodos de avaliação da personalidade comumente usados e que sustentam a ilusão de validade por coeficientes de correlação são os testes e escalas auto-relato (Meyer \& Handler, 1997). Se o critério de validação para uma escala auto-relato for outra escala auto-relato, os resultados desses coeficientes, monométodos, de fato serão altos, mas não necessariamente genuínos coeficientes de validade, embora com freqüência sejam interpretados assim (Meyer, 1996; Meyer \& Handler, 1997). O critério medidas monométodos não detecta diferenciais entre métodos e traços, sendo assim, seus coeficientes de validade serão numericamente maiores do que seriam os coeficientes de validade das medidas heterométodos, favorecendo a impressão de que as primeiras sejam as mais válidas.
Outra questão freqüente nos estudos de validação com o Rorschach e outras técnicas projetivas é o alto número de falsos positivos e negativos quando os critérios adotados são sintomas ou diagnósticos através de questionários que apresentam medidas de psicopatologia. Um exemplo típico é a tentativa de checar a validade e utilidade do instrumento pelo grau de correlação dos seus escores com os critérios e sintomas descritos pela American Psychiatric Association no Manual Diagnóstico e Estatístico de Transtornos Mentais (2002) (Primi, 2005; Franco \& Cardoso, 2005).

O que se constata é que muitos tipos de transtornos psicológicos definem-se em parte por características da personalidade que podem ser apreendidas nas variáveis de técnicas projetivas como o Rorschach, o TAT, o DFH ou outras, e que algumas dessas características podem estar presentes em vários distúrbios caracterológicos ou sintomáticos. No âmbito clínico, o Rorschach volta-se para a descrição de características da personalidade que também podem estar associadas a vários padrões psicopatológicos sem pretensão de fornecer uma medida direta e específica dessas condições (Weiner, 2004). Do mesmo modo, outras técnicas projetivas, como as gráficas ou as temáticas, revelam aspectos da personalidade que se associam e/ou identificam certas patologias, mas que revelam, antes de tudo, características do funcionamento psíquico elucidadas nas teorias psicodinâmicas, enfoque distinto do fenômeno lógico-sintomático descrito nos manuais internacionais de classificação das doenças.

Os informes daí provenientes, essencialmente, podem complementar o diagnóstico identificando (a) traços estáveis; estados afetivos relativamente transitórios e atitudes decorrentes e (b) processos psicológicos latentes que interferem no funcionamento global da personalidade (modos de pensar, sentir e agir). Essas medidas, pouco acessíveis via auto-relato, e que se associam apenas indiretamente com os sintomas descritos no DSM-IV-TR (2002) quarta edição, texto revisado, podem trazer refinamento aos diagnósticos diferenciais de certos transtornos mentais que, embora aparentemente similares, tenham elementos subjacentes contrastantes.

Assim, as correlações tipicamente baixas dos instrumentos de dimensões diferentes não significam que seus informes não possam ser combinados. Medidas válidas não altamente correlacionadas melhoram $\mathrm{o}$ prognóstico de uma variável de critério, em especial, na avaliação funcional da personalidade. O que acontece na prática clínica é que a aplicação de procedimentos multimétodos é determinada justamente pela validade incremental e tais questões são de imediata resolução quando a consideração central for a predição válida de

Psico-USF, v. 11, n. 2, p. 185-193, jul./dez. 2006 
critérios únicos. Se a demanda for a simples predição de resultados, então um instrumento objetivo poderá estar mais próximo desse contexto e os métodos projetivos poderão não revelar vantagens sobre alternativas mais breves e baratas como checklist de sintomas, inventários ou mesmo entrevistas (Meyer \& Archer, 2001).

Como o âmbito clínico requer uma perspectiva diferenciada para as abrangências e limitações dos instrumentos, questões de validade incremental devem centrar-se no mais completo e refinado conhecimento descritivo advindo da eficiência de métodos distintos que possam corroborar uma mesma conclusão, não se tratando da aplicação de um padrão simplista de comparação entre instrumentos para justificar ou não o seu uso (Blais, Hilsenroth, Castelbury, Fowler \& Baity, 2001). E nessa perspectiva, o uso apropriado do Rorschach e demais métodos projetivos não deveria mais incluir estudos de validações que empregam categorias DSM como variáveis dependentes ou então identificar diagnósticos descritos no DSM diretamente nos seus indicadores. $\mathrm{O}$ mais importante na prática clínica e na pesquisa é saber que esses métodos podem identificar de modo mais ou menos acurado forças e fraquezas da personalidade com implicações para planos de tratamento e/ou intervenções, sendo isso especialmente válido para o Rorschach (Weiner, 2000).

Observando-se o paralelismo das dimensões objetivas e projetivas, a segurança dos instrumentos - se confiáveis e válidos - e a competência dos usuários ao avaliarem pessoas para que decisões importantes sejam tomadas, os resultados dos diferentes procedimentos tornam-se uma poderosa fonte multifacetada de informações, mais compreensiva. $\mathrm{E}$ sob tais considerações, serão a seguir abordadas questões específicas relativas aos métodos projetivos mencionados, iniciando-se pelo RCS por concentrar a maior parte dos debates atuais.

Desde sua publicação em 1921 até o final da década de 50, o método de Rorschach foi tido como um procedimento indispensável nos programas de treinamento em psicologia clínica, na área da avaliação da personalidade e dos quadros psicopatológicos. Aí, sob uma ótica de exagerado otimismo - reforçada pelo aval de autores como Klopfer, Holt e Schafer -, despertou grande interesse e tornou-se praticamente sinônimo da psicologia clínica nos EUA (Bornstein, 2001).

Nos anos 60, concomitante à aceitação da abordagem aos testes psicológicos proposta por Cronbach e Meehl enfatizando o paradigma nomotético (em Bornstein, 2001) e do delineamento de Campbell e Fisk (em Bornstein, 2002), intensificavam-se os debates sobre os méritos dos métodos clínicos, bem como as críticas contrárias aos pressupostos básicos e fundamentos empíricos das medidas do Rorschach (Masling, 1997). A isso se somava a má instrumentação e o seu uso indevido por uma parcela significativa de profissionais (Meyer, 1996; Raskin, 2001). Denominações errôneas como "janela para os mistérios da psique humana ou RX da personalidade"; o apelo às interpretações impressionistas e às opiniões teóricas menos atentas à análise cuidadosa dos dados; os registros de estudos iniciais, muito primários e confusos se tomados nos padrões contemporâneos, compuseram o substrato para a fase subseqüente de alvo de tantas críticas (Bornstein, 2001). Como resposta a esse segundo período, marcadamente "opinativo", instalou-se, e com justa razão, uma atitude combativa, tanto por parte dos que criticavam quanto dos que defendiam o instrumento.

Com objetivo de integrar elementos empiricamente sustentáveis, eliminando as personalizações e postulados interpretativos duvidosos, um dos principais proponente do Rorschach, John Exner Jr., publicou em 1974 os resultados iniciais de seus estudos com o instrumento sob o título Um sistema compreensivo, marcando assim um terceiro momento da técnica, de ênfase no rigor psicométrico. A sistematização combinou os elementos sustentáveis dos cinco enfoques utilizados até então nos EUA, porém, com ênfase na abordagem empírica e científica, na padronização e normatização, na administração, pontuação e interpretação dos dados, atingindo com sucesso as inconsistências dos sistemas antecessores.

Entretanto, por essa mesma época, Dawes (1994) publica uma obra bastante provocativa na qual afirma que interpretar um teste de borrões de tinta seria tão científico quanto interpretar sonhos ou cartas. $\mathrm{Na}$ seqüência, Wood, Nezworski e Stejskal (1996) também apresentam severo julgamento especialmente endereçado ao Rorschach no sistema compreensivo, deflagrando assim a atual temporada de ataques. Da série de artigos que criticam ostensivamente o RCS, participam ainda os de Garb, Florio e Grove (1998); Garb, 1999; Garb, Wood, Nesworski, Grove e Stejskal (2001); Hunsley e Baiyle (1999, 2001); Lohr, Fowler e Lilienfeld (2002); Wood, Lilienfeld, Garb e Nezworski (2000 a, 2000b); e Wood, Lilienfeld, Nesworski e Garb (2001).

Para os críticos como Lilienfeld, Wood e Garb (2000); Lohr e colaboradores (2002) e Wood e colaboradores (2001), a volumosa publicação sobre as propriedades psicométricas do RCS ainda mantém seus pontos fundamentais submersos nas mesmas controvérsias das décadas passadas. As principais alegações incluem falta de atualização, referenciais normativos com sérias falhas metodológicas e exclusão de grupos minoritários, o que exacerbaria condições psicopatológicas ou desajustes em quem não os tem. As 
afirmações enganosas também são insistentes na insuficiência dos níveis de confiabilidade e validade para aplicação do RCS no âmbito clínico e na ausência de validade incremental (Lilienfeld e colaboradores, 2000; Wood e colaboradores, 1996).

Indubitavelmente, os instrumentos de avaliação psicológica requerem padronização (material, instrução, procedimentos de aplicação uniformes), apresentação de suas qualidades psicométricas (dados normativos, validades, confiabilidades) e atualizações periódicas. Sendo assim, o RCS vem apresentando, aumentando e revisando suas amostras de não-pacientes, de pacientes, de crianças e adolescentes, no exterior e no Brasil.

Reconhecendo que os dados normativos haviam sido coletados há mais de duas décadas, o sistematizador assumiu um segundo projeto, no qual examinadores experientes analisaram os dados de uma nova amostra de 600 adultos não-pacientes distribuídos por regiões geográficas e parcialmente estratificadas pelo nível socioeconômico (Exner, 2002; Weiner, 2001; 2004). Os resultados preliminares dessa pesquisa compararam as principais variáveis das duas amostras de não-pacientes (Exner, 2001; 2002) e estes mostraram muita semelhança. Esses achados sugerem que os parâmetros normativos estabelecidos anteriormente são representativos dos princípios interpretativos dos quais derivaram, o que desfaz as dúvidas sobre desajustes e exacerbação psicopatológica das suas medidas (Exner, 2002; Weiner, 2004). E no contexto transcultural, as diferenças no RCS, se presentes, poderão refletir, e com exatidão, a apreensão das diversidades culturais, o que não necessariamente significa problemas de validade com o instrumento. Por outra via, a preponderância das evidências na literatura também não conduzem para expectativa de viés étnico no Rorschah (ver Meyer, 2002; Presley, Smith, Hilsenroth \& Exner, 2001; Weiner 2004; Weiner, Spielberg \& Abeles, 2002).

Quanto aos níveis de validade e confiabilidade, estudos e revisões metanalíticas recentes têm mostrado o contrário do alegado pelos críticos. Só nesta década foram feitos quatro estudos metanalíticos originais endereçados à validade do Rorschach e todos concluíram que, em termos gerais, suas validades são sustentáveis por bases empíricas (ver Bornstein, 1996, 1999; Hiller, Rosenthal, Bornstein, Berry \& BrunellNeuleib, 1999; Meyer \& Handler, 1997).

Quanto aos indicadores de confiabilidade, as pesquisas atualizadas comparando amostras de pacientes identificaram coeficiente Kappa para as diversas categorias de codificações no RCS entre 0,79 e 0,88, sendo que 95\% das 164 variáveis do sumário estrutural em duas codificações independentes atenderam a critérios de correlação intraclasse com mediana e média dos coeficientes entre juízes de 0,92 e 0,90 , respectivamente, considerados níveis excelentes para as metodologias estatísticas (ver Acklin, McDowell, Verschell \& Chan, 2000; Meyer, 1996; Meyer \& Handler, 1997; Weiner e colaboradores, 2002). Esses achados reasseguram que as codificações do RCS são confiáveis, o que não exime a confiabilidade dos escores também depender de codificadores hábeis.

A excelente estabilidade temporal no RSC também tem sido apontada como inaceitável para a maioria das variáveis (Garb e colaboradores, 2001; Lilienfeld e col., 2000; Wood \& Lilienfeld, 1999). Cabe destacar que estudos conduzidos por diferentes grupos de pesquisa têm delineamentos peculiares, tais como tamanho da amostra, extensões, intervalos e interveniências nos períodos das reaplicações dificultando a comparação direta entre resultados (Viglione \& Hilsenroth, 2001; Weiner, 2004). A partir de 1993, Exner conduziu muitos estudos direcionados a essa questão. Seus estudos examinaram crianças, adolescentes e adultos em intervalos de retestes, que variaram de alguns dias a oito anos, bem como, o impacto das novas instruções nas respostas dos retestes. A estabilidade temporal foi significativa para adultos, e para crianças, os coeficientes do reteste foram aumentando com o amadurecimento, o que concorda com uma série de resultados já observados em outros estudos não-longitudinais (ver Meyer \& Archer, 2001).

Como fato, quase todas as variáveis relacionadas aos traços característicos codificadas pelo CS mostram importante estabilidade a curto e longo prazo, superando as análises correlacionais recentes 0,75 . Tratando-se especificamente das 19 variáveis centrais, as atuais análises de correlações entre um e três anos são de 0,85 ou acima, o que não deixa dúvidas sobre a confiabilidade temporal dos seus dados (ver Viglione \& Hilsenroth, 2001; Weiner, 2004).

Dados empíricos procedentes de revisões da literatura (Viglione, 1999; Weiner, 2001) e de uma série de exemplos de casos clínicos adequadamente ponderados também apontam evidências positivas de validade incremental nas variáveis do Rorschach sobre outros tipos de testes e informações (ver Stricker \& Gold, 1999; Weiner, 1999).

Particularmente na clínica, os estudos de casos mobilizam questões idiossincráticas que requerem decisões idiográficas, o que demanda escolha de procedimentos adequados aos casos, aos objetivos e às informações relevantes disponíveis por outras fontes. Em virtude disso, na prática clínica, questões relacionadas à validade incremental manifestam-se na utilidade, no impacto dos diferentes instrumentos para tomada de decisões nos casos individuais e nas

Psico-USF, v. 11, n. 2, p. 185-193, jul./dez. 2006 
situações em que haja relutância ou incapacidade para o auto-relato de características da personalidade ou psicopatológicas.

A despeito dos resultados de pesquisa, as técnicas de investigação científica não dispensam contínuos trabalhos de verificação e adaptação à população na qual será aplicada, e estudos normativos com o RCS continuam necessários para aplicações internacionais. Os estudos de adaptação e validação do RCS para a população brasileira têm sido organizados e conduzidos por Nascimento (2004a, b), com destaque para Resultados de estudo normativo para o Sistema Compreensivo do Rorschach: um estudo para a cidade de São Paulo (Nascimento, 2001a) e Método de Rorschach no Sistema Compreensivo: notas sobre estudos brasileiros (Villemor-Amaral, Silva Neto \& Nascimento, 2003).

Desse projeto inicial derivaram ainda vários estudos normativos com considerações especiais aos indicadores de controle e tolerância ao estresse (Nascimento, 2001b); às diferenças socioeconômicas (Nascimento, 2002) e às diferenças educacionais em amostras de não-pacientes (Nascimento, 2004a) e com o confronto dos resultados de uma amostra de paulistanos não-pacientes com os da amostra atualizada de Exner para norte-americanos (Silva Neto, 2004).

$\mathrm{O}$ projeto mais recente está direcionado ao levantamento dos resultados normativos de 200 nãopacientes de diversas cidades do estado de São Paulo para cotejo com os já obtidos para a cidade de São Paulo. Caso sejam compatíveis, serão agregados em uma tabela normativa de 400 não-pacientes para a população do estado de São Paulo (Nascimento, 2004b).

O status de um instrumento de avaliação psicológica determina-se a partir de: (a) suas propriedades psicométricas, (b) propostas presumidas em suas aplicações práticas e (c) freqüência com que é aplicado e valor atribuído pelos usuários. Nesses contextos, apesar do tempo que requer sua aplicação, o Rorschach é um instrumento (a) amplamente usado e muito valorizado por clínicos e pesquisadores de diversos países; (b) bem-estabelecido quanto à validade e confiabilidade dos seus informes na avaliação funcional e dinâmica da personalidade e (c) absolutamente útil no estabelecimento de diagnósticos diferenciais, plano de tratamento e avaliação dos processos interventivos.

Para além de suas propriedades psicométricas, sua função como fonte de projeções, associações e simbolizações idiográficas o enriquece como instrumento diagnóstico. Os estudos de Antunez e Yazigi (2004), Farah (2004) e Villemor-Amaral (2004) exemplificam muito bem o quanto a análise das respostas, modos de expressão e verbalização registra de modo mais sensível certas peculiaridades psicopatológicas em indivíduos, cujas alterações não se manifestaram nos dados psicométricos de forma a distingui-los dos grupos de comparação.

No que se refere aos métodos gráficos ou temáticos, o levantamento da literatura traz um número muito menor de estudos demonstrativos das suas cientificidades mediante quesitos psicométricos. Se especialmente comparados ao RCS, torna-se ainda mais notável a redução dos investimentos nas pesquisas com o DFH e o TAT, o que, conseqüentemente, incita preocupações sobre a falta de sistemas de avaliação e interpretação precisos e confiáveis.

Embora o RCS tenha sido o instrumento projetivo mais duramente atacado, a produção dos estudos em seu conjunto permite expor a inconsistência das argumentações, bem como o interesse políticos das discussões, não só os propriamente científicos. Entretanto, quando estamos diante de críticas feitas a outros métodos de avaliação da personalidade, como é o caso do TAT e do DFH, estas não serão tão diretamente refutáveis, contando-se com restritas publicações de estudos com ambas as técnicas.

Em relação a essas técnicas, Lilienfeld e colaboradores (2000) sinalizam que o problema fundamental está nos esquemas de escores e interpretações baseados em julgamento clínico e que este subestima o rigor técnico e metodológico desses procedimentos.

Ora, o julgamento clínico é fator de incontestável relevância no processo de interpretação e avaliação da personalidade. Autores como Karon (2000) e Tavares (2003) demonstram a importância do raciocínio clínico e o colocam como um prérequisito essencial para compreensão completa e integrada do indivíduo, enfatizando a importância do treinamento clínico supervisionado para a utilização dos métodos projetivos em geral e nos programas de graduação e especialização, o que favorece a integração entre ciência e prática.

Ambos autores demonstram a importância da inter-relação entre a abordagem clínica (idiográfica) e a estatística (nomotética), apontando justamente o problema de que em alguns casos a primeira é subestimada em relação à segunda. $\mathrm{Na}$ verdade, ambas são importantes; uma não deteriora a outra, se complementam, já que as evidências estatísticas empíricas nem sempre contemplam uma larga base de dados representativos da população. No caso da predição clínica, a mesma levará em conta um conjunto de informações globais e integrais sobre o examinando. 
Tavares (2003) discute a "validade clínica", abordando os vértices nomotético e idiográfico no processo de avaliação psicológica e no uso de técnicas específicas para esses fins, enfatizando que "a validade de um instrumento é expressa pelas qualidades ou pelos significados que podem ser associados a ele, e depende da rede de relações que ele estabelece com outros indicadores" (p. 127). O autor ressalta, ainda, que mesmo os instrumentos que são dotados de sofisticados parâmetros psicométricos também devem ser avaliados pela ótica idiográfica. Para Güntert (2000), as perspectivas relacionadas ao geral e ao singular na avaliação da personalidade têm demonstrando que o caráter idiográfico das manifestações psíquicas são tão importantes quanto os nomotéticos, sendo estes mais apreensíveis pelo enfoque clínico que psicométrico.

No que se refere ao DFH, a busca de um sistema de pontuação preciso foi atingida no estudo de Naglieri (1988), nos Estados Unidos, que obteve índices de precisão por consistência interna que variaram de 0,83 a 0,89 , por teste-reteste entre 0,60 e 0,89 e entre avaliadores com variações de 0,86 a 0,89 . No Brasil encontram-se os estudos de Marques, Pasian, Franco, Panosso, Viana e Oliveira (2002) e de Wechsler (2003), ambos com bons resultados sobre a precisão dos sistemas de pontuação propostos por Goodenough (1926), Harris (1991) e Naglieri (1988). A validade desse teste foi verificada em nosso meio por autores como Bandeira e Hutz (1994); Sisto (2000) e Wechsler (2003), com resultados muito satisfatórios sobre a confiabilidade no uso do método de acordo com os parâmetros psicométricos.

No caso do TAT, não foram encontrados trabalhos que visassem à elaboração de um sistema mais preciso de análise das respostas que o proposto por Murray $(1943,1995)$ e a Escala de Relações Objetais e Cognição Social (SCORS) proposta por Western, Lohr, Silk, Keber e Goodrich (em Ackerman, Hilsenroth, Clemence, Weatherill \& Fowler, 2001). Ainda assim, cabe ressaltar duas interessantes publicações.

A primeira determina a possibilidade de se chegar a níveis aceitáveis de consistência interna (coeficiente alfa $>0,7$ ) para quatro medidas da Escala de Relações Objetais e Cognição Social de Western e colaboradores (1989) e do TAT quando considerados itens escalares (ver Hibbard, Mitchell \& Porcerelli, 2001). A outra publicação amplia os achados dessa pesquisa prévia, demonstrando a confiabilidade e a validade convergente entre o Rorschach Mutuality of Autonomy Scale - MOA (Urist, 1977, em Ackerman e cols., 2001) e a SCORS (Westen, 1995, em Ackerman e cols., 2001) como medidas de funcionamento interpessoa. Mais especificamente, enfoca a validade convergente entre as medidas da Escala Rorschach MOA e oito medidas para variáveis da SCORS nas narrativas do TAT (complexidade; afeto; investimento emocional nos relacionamentos, investimento emocional em valores morais; compreensão social; manejo dos impulsos/agressividade, auto-estima; identidade/ coe-rência do self).

Como conclusão, o estudo apóia o uso dessas medidas do Rorschach e TAT para avaliação das relações objetais em amostras de pacientes. Quanto às evidências de validade, em parte também foram alcançadas nos estudos que investigaram problemas específicos com delineamentos de pesquisa comparativa entre desempenhos de grupos contrastantes, com base em critérios externos. Todavia, se o critério de cientificidade tiver como base única os parâmetros da psicometria, o TAT não se apresentará tão próximo, embora sob a ótica do raciocínio clínico e dos estudo dos aspectos idiográficos, o conjunto dos seus informes sobre a pessoa e o seu valor não deva ser negligenciado.

Finalizando, até certo ponto o rigor psicométrico garante confiabilidade aos instrumentos projetivos, é um quesito necessário, e as investigações devem continuar na direção dos procedimentos metodológicos que acrescentem evidências de validade às produzidas até então. Por outro lado, a validade do enfoque clínico nas interpretações dos resultados, correlacionada a outros dados provenientes de várias fontes sobre o mesmo indivíduo é condizente com a complexidade da natureza humana, com a busca da compreensão global e da dinâmica da sua singularidade e com uma via mais eficaz para propostas interventivas nos vários campos de atuação do psicólogo.

\section{Referências}

Ackerman, S. J., Hilsenroth, M. J., Clemence, A. J., Weatherill, R. \& Fowler, J. C. (2001). Convergent validity of Rorshach and TAT Scales of Object Relations. Journal of Personality Assessment, 77(2), 295306.

Acklin, M. W., McDowell, C. J., Verschell, M. S. \& Chan, D. (2000). Interobserver agreement, intraobserver agreement, and the Rorschach Comprehensive Sistem. Journal of Personality Assessment, 74, 15-57.

Antunez, A. E. A. \& Yazigi, L. (2004). Análise fenomenológica estrutural do Rorschach de pacientes com transtorno obsessivo-compulsivo. Em C. E. Vaz e R. L. Graeff: Técnicas projetivas: produtividade em pesquisa. Porto Alegre: SBRo. 
American Psychiatric Association (2002). Manual diagnóstico e estatístico de transtornos mentais (4 ed. rev.). Porto Alegre: Artmed.

Bandeira, D. R \& Hutz, C. S. (1994). A contribuição dos testes DFH, Bender e Raven na predição do rendimento escolar na primeira série. Psicologia: Teoria e Prática, 10 (1), 59-72.

Blais, M. A., Hilsenroth, M. J., Castelbury, F., Fowler, J. C. \& Baity, M. R. (2001). Predicting DSM-IV Cluster $B$ personality disorder criteria from MMPI-2 and Rorschach data: A test of incremental validity. Journal of Personality Assessment, 76 150-168.

Bornstein, R. F. (1996). Construct validity of the Rorschach Oral Dependency Scale: 1967-1995. Psychological Assessment, 8, 200-505.

Bornstein, R. F. (1999). Criterion validity of objective and projective dependency: A meta-analitic assessment of behavioral prediction. Psychological Assessment, 11, 48-57.

Bornstein, R. F. (2001). Clinical utility of the Rorschach Inkblot Method: Reframing the debate. Journal of Personality Assessment, 77 (1), 39-47.

Bornstein, R. F. (2002). A process dissociation approach to objective-projective test score interrelationship. Journal of Personality Assessment, 78 (1), 47-68.

Dawes, R. M. (1994). House of cards: psychology and psychoterapy built on mith. New York: Free Press.

Exner, J. R. (1999). Em E. Embrtson \& S. L. Hershhberger The Rorschach: Measurement concepts and issues of validity. The new rules of mesurement: What every psychologist and educator should know. Mahwah, NJ: Erlbaum.

Exner, J. E., Jr. (1991). A Rorschach workbook for the comprehensive system. $V$ 2: Interpretation (2nd ed.). New York: John Wiley \& Sons, Inc.

Exner, J. E., Jr. (1994). El Rorschach: un sistema comprehensivo. $V$ 1: fundamentos basicos (3rd ed.). Madrid: Prismática Rorschach Workshops.

Exner, J. E., Jr. (2001). A Rorschach workbook for the comprehensive system (5th ed.). Asheville, NC: Rorschach Workshops.

Exner, J. E., Jr. (2002). A new nonpacient sample for the Rorschach Comprehensive System: A progress report. Journal of Personality Assessment, 78(3), 391-404.

Exner, J. E., Jr. (2003). The Rorschach: A comprehensive system. Volume 1: Basic foundations and principles of interpretation (4th ed.). Hoboken: Wiley.
Farah, F. H. Z. O transtorno do pânico no método de Rorschach e na Escala de Ansiedade de Beck (2004). (Dissertação de mestrado). Universidade São Francisco.

Franco, R. R. C. \& Cardoso, L. M. (2005) A Psicopatologia no Teste de Pfister. Em A. E. Villemor-Amaral (2005). O Teste das Pirâmides de Pfister. São Paulo: CETEPP.

Garb, H. N. (1999). Call for a moratorium on the use of de Rorschach Inklot Test in clinical e forensic setting. Assessment, 6, 313-315.

Garb, H. N., Florio, C. M. \& Grove, W. M. (1998). The validity of the Rorschach and Minnesota Multiphasic Personality Inventory: Results from meta-analyses. Psychological Science, 9, 402-404.

Garb, H. N., Wood, J. M., Nezworski, M. T., Grove, W. M. \& Stejskal, W. J. (2001). Toward a resolution of the Rorschach Controversy. Psychological Assessment, 13, 433-448.

Goodenough, F. L. (1926). Measurement of intelligence by drawings. World Book Company, The House of Applied Knowledge, New York.

Güntert, A. E. V. A. (2000). Técnicas projetivas: o geral e o singular em avaliação psicológica. Em F. F. Sisto, E. T. B Sbardelini \& R. Primi (Orgs.). Contextos e questões em avaliação psicológica (pp. 77-84). São Paulo: Casa do Psicólogo.

Harris, D. B. (1991). El test de Goodenough. Revisión, ampliación y actualización. Espanha: Paidós.

Herzberg, E. \& Mattar, A (2005, julho) Psychological examination techniques and resources used in the Department of Clinical Psychology of the University of São Paulo. Trabalho apresentado no XVIII Congresso Internacional de Rorschach e Métodos Projetivos.

Hibbard, S., Mitchell, D. \& Porcerrelli, J. (2001). Internal consistency of the object relations and Social Scales for the Thematic Apperception Test. Journal of Persoanlity Assessment, 77(3), 408-419.

Hiller, J. B., Rosenthal, R., Bornstein, R. F., Berry, D. T. R. \& Brunell-Neuleib, S. (1999). A comparative metaanalysis of Rorschach and MMPI validity. Psychological Assessment, 11, 278-296.

Hunsley, J. \& Bailey, J. M. (1999). The clinical validity of the Rorschach: Unfulfilled promises and an certain future. Psychological Assessment, 11, 266-277.

Hunsley, J. \& Bailey, J. M. (2001). Whither the Rorschach? An analysis of the evidence. Psychological Assessment, 13, 472-485. 
Karon, B. P. (2000). The clinical interpretation of the Thematic Apperception Test, Rorschach, and other clinical data: A reexamination of statistical versus clinical prediction. Professional Psychology: Research and Practice. 31(2) 230-233.

Lilienfeld, S. O, Wood, J. M., \& Garb, H. N. (2000). The scientific status of projective techniques. Psychological Science in the Public Interest, 1, 27-66.

Lohr, J. M., Fowler, K. A. \& Lilienfeld, S. O. (2002). The dissimination and promotion of pseudocience in clinical psychology: The challenge to legitimate clinical science. Clinical Psychologist, 55, 4-10.

Machover, K. (1949). Personality projection in the drawing of the buman figure: A method of personality investigation. Springfield: C. C. Thomas.

Marques, S. L., Pasian, S. R., Franco, M. A. P., Panosso, I. R., Viana \& Oliveira, D. A. (2002). Fidedignidade do sistema de Goodenough de avaliação cognitiva: uma visão do contexto atual. Estudo de Psicologia, 7(1), 57-64.

Masling, J. (1997). On the nature and utility of the projective tests and objective tests. Journal of Personality Assessment, 69(2), 257-270.

Masling, J. (2002) How do I score thee? Let me count the ways. Or some different methods of caracterizing Rorschach responses. Journal of Personality Assessment, 79(3), 399-421.

Meyer, G. J. (1996). Construct validation of scales derived from the Rorschach Method: A review of issues and introduction to Rorschach Rating Scale. Journal of Personality Assessment, 67(3), 598-628.

Meyer, G. J. (2002). Exploring possible ethnic differences and bias in the Rorschach Comprehensive System. Journal of Personality Assessment, 78(1), 104-129.

Meyer, G. J. \& Handler, L. (1997). The ability of the Rorschach to predict subsequent outcome: Metaanalysis of the Rorschach Prognostic Rating Scale. Journal of Personality Assessment, 69, 1-38.

Meyer, G. J. \& Archer, R. P. (2001). Hard science of Rorschach research: What do we know and where do we go? Psychological Assessment, 13(4), 486-502.

Murray, H. A. (1995). Teste de Apercepção Temática: TAT (2a ed.). São Paulo: Casa do Psicólogo. (Trabalho original publicado em 1943).

Naglieri, J. A. (1988). Draw a person: A quantitative scoring system. Manual. The Psychological Corporation Harcourt Brace Jovanovich, Inc.

Nascimento, R. S. G. F. do (2001a). Resultados de estudo normativo para o Sistema Compreensivo de Rorschach: um estudo para a cidade de São Paulo. Comunicação apresentada no The International Rorschach Society's Summers Seminars and Dialogues, Spiez, Suíça.

Nascimento, R. S. G. F. do (2001b). Estudo normativo do Sistema Compreensivo do Rorschach para a cidade de São Paulo: uma atenção especial para o controle e tolerância ao estresse. Comunicação apresentada no V Congresso Nacional de Psicodiagnóstico e XII Jornadas Nacionales de A . D. E. I. P. La Plata, Argentina.

Nascimento, R. S. G. F do (2002). Estudio normativo para el Sistema Comprehensivo del Rorschach en la ciudad de San Pablo: consideraciones en las diferencias socioeconómicas. Trabalho apresentado no simpósio: Realidades Culturales Iberoamericanas a Confrontar em Muestras de Non-Pacientes, XVII Congresso da Internacional Rorschach Society, Roma, Itália.

Nascimento, R. S. G. F do (2004a). The impact of education and/or socioeconomic conditions on Rorschach data in a brasilian nonpatient sample. Rorschachiana, 26, 45-62.

Nascimento, R. S. G. F. do (2004b) Um estudo normativo do Rorschach para cidades do estado de São Paulo: Resultados parciais. Em C. E. Vaz \& R. L. Graeff (Orgs.). Trabalhos completos III Congresso Nacional da Sociedade Brasileira de Rorschach e Métodos Projetivos (pp. 27-34). Porto Alegre: SBRo.

Preley, G., Smith, C., Hilsenroth, M. \& Exner, J. (2001). Clinical utility of the Rorschach with African Americans. Journal of Personality Assessment, 77(3), 491507.

Primi, R. (2005). Evidências de validade. Em A. E. Villemor-Amaral (2005). O Teste das Pirâmides de Pfister. São Paulo: CETEPP.

Raskin, J. D. (2001). Construtivism and the projective assessment of meaning in Rorschach administration. Journal of Personality Assessment, 77(1), 139-161.

Silva, A. C. P. Neto (2004). Usando corretamente o Sistema Compreensivo do Rorschach em indivíduos brasileiros. Em C. E. Vaz \& R. L. Graeff (Orgs.). Trabalhos completos III Congresso Nacional da Sociedade Brasileira de Rorschach e Métodos Projetivos (pp. 43-47). Porto Alegre: SBRo.

Sisto, F. F. (2000). Relationships of the piagetian cognitive development to Human Figure Drawing. Journal of School Psychology, 30(4), 432-441.

Stricker, G. \& Gold, J. R. (1999). The Rorschach: Toward a nomothetically based, idiographically applicable configurational model. Psychological Assessment, 11, 240-250. 
Tavares, M. (2003). Validade clínica. Psico-USF, 8(2), $125-$ 136, julho/dezembro.

Villemor-Amaral, A. E. (2004). Análise fenômenoestrutural e análise simbólica: duas abordagens complementares numa perspectiva qualitativa. Em C. E. Vaz \& R. L. Graeff. Técnicas projetivas: produtividade em pesquisa. Porto Alegre: SBRo.

Villemor-Amaral, A. E., Silva, Neto, A. C. P. \& Nascimento, R. S. G. F. (2003). O método de Rorschach no Sistema Compreensivo: notas sobre estudos brasileiros (v. 1). São Paulo: Casa do Psicólogo.

Viglione, D. J. (1999). A review of recent research addressing the utility of the Rorschach. Psychological Assessment, 11, 251-265.

Viglione, D. J. \& Hilsenroth, M. J. (2001). The Rorschach: Facts, fiction, and future. Psychological Assessment, 13, 452-471.

Wechsler, S. M. (2003). O desenho da figura humana: avaliação do desenvolvimento cognitivo de crianças brasileiras. $3^{\mathrm{a}}$. ed. Campinas: Impressão Digital do Brasil.

Weiner, I. B. (1999). What the Rorschach can do for you: Incremental validity in clinical applications. Assessment, 6, 327-338.

Weiner, I. B. (2000). Using the Rorschach properly in practice and research. Journal of Clinical Psychology, 56 (3), 435-438.

Weiner, I. B. (2001). Advancing the science of psychological assessment: The Rorschach Inkblot Method as exemplar. Psychological Assessment, 13, 423432.

Weiner, I. B. (2004). Utility of Rorschach assessment in clinical and forensic practice. Special Feature Articles. (Ovid Full Text Bibliographic Links).
Weiner, I. B., Spielberger, C. H. \& Abeles, N. (2002). Scientific psychology and the Rorschach Inkblot Method. The Clinical Psychologist, 55, 7-12.

Western, D., Lohr, N., Silk, K. Kerber, K. \& Goodrich, S. (1989). Object relations and social cognition TAT scoring manual (4 ${ }^{\text {th }}$ ed.). Unpublished manuscript, University of Michigan, Ann Harbor. (em Journal of Personality Assessment, 77(2), 295-306).

Wood, J. M. \& Lilienfeld, S. O. (1999). The Rorschach Inkblot Tests: A case of overstatement? Assessment, 6, 341-349.

Wood, J. M., Nezworski, M. T. \& Stejskal, W. J. (1996). The Comprehensive System for the Rorschach: A critical examination. Psychological Science, 7, 3-10.

Wood, J. M., Lilienfeld, S. O., Garb, H. N. \& Nezworski, M. T. (2000a). The Rorschach test in clinical diagnosis: A critical review, with a backward look at Garfield (1947). Journal of Clinical Psychology, 56, 395-430.

Wood, J. M., Lilienfeld, S. O., Garb, H. N. \& Nezworski, M. T (2000b). Limitations of the Rorschach as a diagnostic tool: A reply to Garfield (2000), Lerner (2000), and Weiner (2000). Journal of Clinical Psychology, $56,441-448$.

Wood, J. M., Lilienfeld, S. O., Nezworski, M. T \& Garb, H. N. (2001). Coming to grips with negative evidence for the Comprehensive System for the Rorschach: A comment on Gacono, Loving, and Bolholt; Ganelle; and Bornstein. Journal of Personality Assessment, 77 (1), 48-70.

Recebido em abril de 2006 Aprovado em setembro de 2006

Sobre as autoras:

Anna Elisa de Villemor-Amaral é mestre e doutora pela UNIFESP/EPM, com pós-doutorado pela Universidade da Savóia, na França. É professora do programa de Pós-Graduação em Psicologia da Universidade São Francisco e professora da Faculdade de Psicologia da PUC-SP.

Lílian Pasqualini-Casado é psicóloga pela Universidade São Marcos, tem especialização no Método de Rorschach pela Sociedade Rorschach de São Paulo e Instituto de Psicologia Aplicada e Formação - Lisboa e é mestranda pela Universidade São Francisco, Itatiba, SP. 
